\title{
Craneofaringioma en pediatría: Diagnóstico y tratamiento
}

\section{Craniopharyngioma in pediatrics: diagnosis and treatment}

\author{
"Edgar Cabrera-Bernal MD Esp1* \\ ${ }^{1}$ Fundación Hospital Pediátrico la Misericordia, Bogotá - Colombia.
}

Recibido el 30 de diciembre de 2019; Aceptado el 26 de marzo de 2020

\section{Resumen}

Los craneofaringiomas (CF) son tumores raros de baja malignidad histológica, que surgen a lo largo del conducto craneofaríngeo. Los dos subtipos histológicos, craneofaringioma adamantinomatoso (CFA) y papilar (CFP), difieren en su origen y distribución por edad. Los CFA se diagnostican con un pico de incidencia bimodal (5-15 años y 45-60 años), mientras que los CFP se presentan exclusivamente en adultos, principalmente en la quinta y sexta décadas de la vida. Los CFA son generados por mutaciones somáticas en CTNNB1, codificador de $\beta$-catenina y son predominantemente de aspecto quístico. Los CFP albergan mutaciones somáticas en BRAF V600E y son típicamente tumores sólidos. Las manifestaciones clínicas de hipertensión endocraneana, compromiso visual o deficiencias endocrinas, sirven de alerta para solicitar imágenes. El tratamiento comprende generalmente neurocirugía y radioterapia (RT); la quimioterapia intraquística se usa en el CFA monoquístico. Aunque la supervivencia a largo plazo es alta, con frecuencia se ven afectadas la calidad de vida y la función neuropsicológica, debido a la proximidad anatómica al quiasma óptico, el hipotálamo y la glándula pituitaria. La afectación hipotalámica por el tumor y las lesiones secundarias al tratamiento, con frecuencia resultan en obesidad hipotalámica y déficits neuropsicosociales.

Palabras clave: Craneofaringioma; pediatría; clasificación; fisiopatología; diagnóstico, diagnóstico por imagen; cirugía; radioterapia; tratamiento farmacológico.

\section{Abstract}

Surgery is the main treatment modality for non-metastatic melanoma, and resectable metastatic melanoCraniopharyngiomas (CP) are rare tumors of low histological malignancy that arise along the craniopharyngeal duct. The two histological subtypes, adamantinomatous craniopharyngioma (ACP) and papillary (PCP), differ in origin and distribution by age. ACPs are diagnosed with a bimodal incidence peak (5-15 years and 45-60 years), while PCPs occur exclusively in adults, mainly in the fifth and sixth decades of life. ACPs are generated by somatic mutations in CTNNB1, encoder of $\beta$-catenin and are predominantly cystic in appearance. PCPs have somatic mutations in BRAF $\mathrm{V} 600 \mathrm{E}$ and are typically solid tumors. The clinical manifestations of endocranial hypertension, visual compromise or endocrine deficiencies should alert us to request images. The treatment generally includes neurosurgery and radiotherapy (RT); Intracystic chemotherapy is used in monocystic ACP. Although long-term survival is high, quality of life and neuropsychological function are frequently

\footnotetext{
* Autor para correspondencia: Edgar Cabrera-Bernal. Médico Cirujano, Oncohematólogo Pediatra. Hospital Pediátrico La Misericordia. Correo electrónico: edgarcabrerabernal@gmail.com 
affected, due to the anatomical proximity to the optic chiasma, the hypothalamus and the pituitary gland. Hypothalamic involvement by the tumor and injuries secondary to treatment often result in hypothalamic obesity and neuropsicosocial deficits.

Keywords: Craniopharyngioma; pediatrics; classification; physiopathology; diagnosis; diagnostic imaging; surgery; radiotherapy; drug therapy.

\section{Introducción}

Los craneofaringiomas (CF) son tumores poco frecuentes; surgen de los remanentes ectodérmicos de la hendidura de Rathke o de otro epitelio embrionario. Su histología es de bajo grado (WHO I) y se localiza con frecuencia en el área selar y paraselar ${ }^{1}$. El tratamiento del CF puede incluir una combinación de cirugía, radioterapia, drenaje quístico o interferón a (IFNa) intraquístico. El tratamiento del craneofaringioma recidivante depende del tratamiento inicial. La supervivencia global a 20 años es alta, entre 87 al $95 \%$, sin embargo, la calidad de vida a largo plazo con frecuencia se ve afectada debido a las secuelas causadas por la proximidad del tumor al eje hipotálamo-hipófisis y al quiasma óptico, además de los efectos secundarios del tratamiento ${ }^{2}$.

Esta revisión pretende dar una visión general de la fisiopatología, diagnóstico, tratamiento y secuelas del CF en pediatría, con el fin de actualizar el abordaje de esta patología.

Se realizó una búsqueda de la literatura con los respectivos terminos Mesh, para proceder a establecer estrategias de búsqueda. Las búsquedas se hicieron en Medline (PubMed), Embase, Google académico y Lilacs. Se limitaron los artículos a los idiomas inglés y español y no hubo restricciones por fecha de publicación.

\section{Epidemiología}

Los craneofaringiomas son tumores poco frecuentes, con una incidencia 0.13 a 2 casos por 100.000 personas/año, representando cerca del 5 al $11 \%$ de todos los tumores intracraneales en pediatría y el $50 \%$ de los tumores selares y paraselares 1-7. Cerca del 30 al 50 \% son diagnosticados durante la infancia y la adolescencia ${ }^{3}$.

Hay dos subtipos histológicos, craneofarin- gioma adamantinomatoso (CFA) y papilar (CFP). El CFA tiene una presentación bimodal: un primer pico de incidencia entre los 5 y 15 años y un segundo pico en edad adulta, entre 45 y 60 años ${ }^{5,8}$.

En la edad pediátrica, el CFA con quistes es el más frecuente ${ }^{9}$. Se han reportado muy pocos casos en fetos y en neonatos ${ }^{10-12}$.

El CFP es prácticamente exclusivo de adultos, con una edad promedio de presentación entre 40 y 55 años ${ }^{13}$; en estudios poblacionales no se ha evidenciado predilección por género o raza $3,14,15$.

Se han descrito casos en una misma familia $^{16,17}$, pero no se ha identificado alguna susceptibilidad genética o factores predisponentes.

\section{Supervivencia y morbilidad}

Los estudios han reportado que, en promedio, la mortalidad global en el CF es de tres a cinco veces mayor que la observada en la población general ${ }^{18}$. La supervivencia global descrita en cohortes pediátricas varía del $83 \%$ al $96 \%$ a los 5 años ${ }^{19}$, del $65 \%$ al $100 \%$ a los 10 años ${ }^{20,21} \mathrm{y}$ del $62 \%$ a los 20 años.

La morbilidad a largo plazo está asociada con factores del comportamiento del tumor o por el tratamiento, tales como enfermedad progresiva con recurrencias múltiples, enfermedad cerebrovascular, como dilataciones fusiformes de la arteria carótida ${ }^{22}$ y deficiencias neuroendocrinas crónicas ${ }^{20,21,23-25}$. En el CF se ha informado hígado graso no alcohólico, que conduce a cirrosis hepática, secundaria a obesidad hipotalámica mórbida ${ }^{20,21,26-28}$.

Los pacientes con CF tienen una tasa de mortalidad aumentada de 3 a 19 veces más que la población general, por enfermedad cardiovascular asociada con sindrome metabólico. Se 
observa una tasa de riesgo cardiovascular mayor en mujeres con CF potencialmente causada por deficiencia de estrógenos debido a hipogonadismo central ${ }^{29}$.

\section{Patología y genética molecular}

Los CF se clasifican en dos subtipos: CFA y CFP; los CFA en el $95 \%$ muestran mutaciones somáticas en el exón 3 del gen de la proteína $\beta$-catenina (CTNNB1) que afectan los residuos reguladores involucrados en la estabilidad de la proteína ${ }^{30-32}$. La consecuencia de estas mutaciones es que la $\beta$-catenina no se puede degradar de manera eficiente, se acumula dentro de la célula, y sobreactiva la vía WNT / $\beta$-catenina, una vía crítica tanto en la fisiología normal, como en la enfermedad, incluyendo el cáncer ${ }^{33,34}$. Los análisis de modelos murinos han revelado que los grupos de células que acumulan $\beta$-catenina, actúan como "centros" de señalización dentro del tumor. Estos grupos secretan una gran cantidad de factores de crecimiento y citoquinas, incluyendo sonic hedgehog $(\mathrm{SHH})$, citoquinas como IL-1 e IL-6 y factores de crecimiento, como factor de crecimiento epidérmico, factor de crecimiento de fibroblastos, WNT, factor de crecimiento transformante $\beta$ y proteínas morfogenéticas óseas, entre otros, que activan vías específicas en las células tumorales circundantes cercanas ${ }^{1,35-37}$.

Los CFP también tienen baja tasa de mutaciones (15 mutaciones por megabase). Hasta el momento sólo se han detectado las mutaciones somáticas en BRAFV600E, cuya expresión se ha observado en la gran mayoría de las células tumorales $32,38,39$. El BRAF es un regulador de la vía MAPK, que controla muchos procesos fisiológicos y frecuentemente está disregulada en cáncer. EN CFP la activación de la vía MAPK se limita a unas pocas células tumorales basales que rodean los núcleos fibrovasculares, que son estructuras que contienen estroma y vasos sanguíneos rodeados por un epitelio de revestimiento que sustenta el crecimiento tumoral ${ }^{39,40}$.

Con base en estos recientes hallazgos en genética molecular, la terapia dirigida ha proporcionado nuevas perspectivas prometedoras para el tratamiento de CFP que albergan mutaciones BRAF-V600E, detectadas exclusivamente en la CFP de inicio en la edad adulta ${ }^{2}$.

\section{Diagnóstico}

\section{Cuadro Clínico}

El diagnóstico de CF en pediatría generalmente es tardío, se realiza años después de la aparición de los síntomas ${ }^{41}$. En un estudio se evidenció que la mediana de duración de los síntomas antes del diagnóstico fue de seis meses con un rango de 0.1 a 108 meses ${ }^{42}$. El cuadro clínico es caracterizado por hipertensión endocraneana (cefalea, náuseas, vómito) en el momento del diagnóstico.

Los CF, por su localización selar/paraselar, pueden afectar la función endocrina evidenciada en el 52 a $87 \%$ de los casos, y por su cercanía con el quiasma y nervios ópticos, puede generar déficit visual, reportado en 62 al $84 \%$ de los pacientes ${ }^{43,44}$. En algunos pacientes el CF puede progresar y crecer con extensión al tercer ventrículo y generar hidrocefalia. Es muy raro que se extienda a fosa posterior y en este caso, se puede manifestar con ataxia, cefalea, diplopía o hipoacusia ${ }^{45}$.

Las deficiencias endocrinas son frecuentemente causadas por el compromiso del tumor del eje hipotálamo-hipofisario o secundarias al tratamiento y pueden afectar la secreción de la hormona de crecimiento (75\% de los pacientes), hormonas estimulantes gonodotropas (40\% de los pacientes), hormona estimulante de la tiroides ( $25 \%$ de los pacientes), hormona adrenocorticotrópica ( $25 \%$ de los pacientes) o manifestarse con diabetes insípida (17 al $27 \%$ de los pacientes). Los déficits endocrinos son la primera manifestación clínica en el 40-87 \% de los pacientes diagnosticados con CF ${ }^{46-48}$. Entre de las manifestaciones clínicas también se han evidenciado tasas de crecimiento anormalmente bajas ${ }^{41}$.

Aunque la obesidad hipotalámica es una secuela frecuente en el CFA de inicio en la infancia ${ }^{49}$, el síndrome diencefálico, que conduce a la pérdida de peso severa y la caquexia, también puede ocurrir como un trastorno hi- 
potalámico, como en el observado en el $4.3 \%$ de 485 pacientes reportados en el Registro Alemán de Craneofaringioma Infantil 50,51.

Una combinación de los síntomas de cefalea, deterioro visual, retraso del crecimiento y/o poliuria/polidipsia es altamente indicativa de CF y la conducta es realizar estudios de neuroimágenes ${ }^{2}$.

\section{Imágenes}

La localización más común del CF es supraselar con un componente intraselar menor ${ }^{1}$. Aproximadamente el $20 \%$ de los tumores son exclusivamente supraselares y $5 \%$ exclusivamente intraselares ${ }^{44}$. El CFP se caracteriza porque generalmente no está calcificado y tiene un componente primordialmente sólido, mientras que para el CFA, sus características radiológicas se pueden resumir en la llamada regla del $90 \%$ : aproximadamente el $90 \%$ son predominantemente quísticos, el $90 \%$ muestra calcificaciones y $90 \%$ resalta con contraste en las paredes del quiste $^{2}$. En la resonancia magnética $(R M)$ sin contraste, las partes sólidas y las paredes del quiste pueden mostrar una variedad de señales en T1. En las imágenes en T2, los tumores suelen ser hipo e hiperintensos ${ }^{52}$. Esta variabilidad de la señal se debe a la distribución no homogénea de partes calcificadas y a la variación de las señales de las calcificaciones en la RM. Es por esto que, evaluar calcificaciones en el CF, generalmente no es posible con la RM. Las secuencias ideales para la identificación de calcificaciones son $\mathrm{T} 2$ o secuencias ponderadas por susceptibilidad (SWI), las cuales se ven obstaculizadas por el contenido de aire de los senos paranasales ${ }^{2}$. Por lo tanto, la tomografía computarizada (TC) sigue siendo el estándar de oro para la identificación de calcificaciones en esta área. La prueba de calcificaciones en imágenes es importante para el diagnóstico diferencial de otras masas selares y para la detección de pequeños restos postoperatorios después de la resección indetectable en la RM.

Hay pocos reportes en la literatura de $\mathrm{CP}$ con metástasis espinales $53 \mathrm{y}$ de ubicación ectópica, observados a lo largo del sitio quirúrgico ${ }^{54}$.Losprincipalesdiagnósticosdiferen- ciales de masas selares/paraselares; en niños son los gliomas de bajo grado y los tumores de células germinales ${ }^{55}$.

Las imágenes de rutina durante el seguimiento podrían limitarse a la RM sin contraste, para disminuir el riesgo potencial de depósitos de gadolinio.

\section{Tratamiento}

\section{Cirugía}

El manejo quirúrgico, especialmente en niños, sigue siendo controvertido. El mejor tratamiento para el CF es aquel que genere menor morbilidad a largo plazo. El tratamiento puede incluir sólo cirugía o sólo radioterapia (RT), pero más frecuentemente es una combinación de las dos. En los casos de sólo cirugía, implica una resección radical y sería adecuada para tumores que pueden resecarse completamente sin lesión neurovascular y discapacidad visual ${ }^{2,56}$.

Evitar el daño hipotalámico irreversible es un objetivo clave en el tratamiento del CF y, para esto, la cirugía limitada y la RT son apropiadas para la mayoría de los pacientes, incluidos aquellos en quienes podría considerarse la cirugía radical. El concepto de cirugía limitada y RT, implica el uso de cirugía para prevenir y aliviar los síntomas y mejorar la la respuesta a la RT ${ }^{57}$. La cirugía limitada puede incluir la resección parcial, la fenestración o aspiración del quiste, la colocación del catéter-reservorio de Ommaya o la derivación del líquido cefalorraquídeo (LCR ${ }^{2}$.

\section{Terapias intraquísticas}

Los tratamientos intraquísticos son una opción a la resección quirúrgica en pacientes bien seleccionados con CFA puro o principalmente monoquístico. Es particularmente útil en los pacientes más jóvenes, y pueden ayudar a posponer la RT; deben ser realizados por equipos multidisciplinarios experimentados.

El catéter de Ommaya se puede utilizar para descomprimir la porción quística de manera repetida y, además, para la instilación de sustancias esclerosantes.

El tratamiento con IFNa proporciona la mejor relación beneficio-riesgo, pero se limita a la 
porción quística, sin ningún efecto sobre el componente sólido del tumor. Una revisión internacional reciente de 56 niños tratados con IFNa, mostró progresión en 42 pacientes después de un tiempo medio de 14 meses ${ }^{58,59}$.

También se han usado la instilación por via intraquística de isótopos radioactivos como ${ }^{90}$ Ytrium y ${ }^{32}$ Phosphorus, o quimioterapia como la bleomicina, pero estos agentes pueden estar asociados con neurotoxicidad irreversible o incluso la muerte, y no han demostrado ser consistentemente eficaces. De hecho, en una revisión reciente, la evidencia disponible no pudo soportar el uso de bleomicina intraquística en niños sobre la base de los beneficios y los efectos secundarios ${ }^{60}$. Hasta ahora los estudios sobre terapias intraquísticas son pequeños y de baja potencia, con datos limitados para respaldar su uso ${ }^{2}$.

\section{Radioterapia (RT)}

La RT puede ser de fotones o protones; en la mayoría de los casos, se administra en el momento de la progresión después de una cirugía previa ${ }^{61}$. En el primer escenario se realizó una resección radical y se presenta una recidiva inesperada. En el segundo escenario se realiza una resección parcial, se hace seguimiento clínico y de imágenes hasta evidenciar progresión. En este escenario es importante tener en cuenta que la extensión de la resección quirúrgica se puede minimizar a la cantidad requerida con la menor morbilidad, porque no se ha demostrado que la cantidad de tumor residual influya en el resultado después de la $R T{ }^{57}$.

El CF es uno de los tumores cerebrales pediátricos para los que la terapia de protones es muy importante. La RT convencional utiliza rayos $\mathrm{X}$ dirigidos desde múltiples direcciones para ajustar la dosis de prescripción al volumen objetivo, a expensas del tejido no objetivo que recibe una dosis colateral asociada con la entrada y salida de los haces transversales. La terapia de protones despliega menos haces que depositan dosis a lo largo de un camino que termina en el objetivo. La dosis al tejido no objetivo se reduce significativamente.

Independientemente de la modalidad, en la mayoría de los casos el eje hipotalámico-hipofisario, el quiasma óptico y los nervios ópticos, los componentes principales de la circulación cerebral y algunas regiones del tronco encefálico, reciben también la dosis prescrita. Esto deja los efectos cognitivos como uno de los pocos resultados funcionales que podrían justificar la ventaja de la RT de protones sobre la de fotones $^{62}$. En 2011, se inició un estudio prospectivo con terapia de protones dispersos pasivamente (primera generación), el cual reclutó un total de 94 participantes de o a 21 años hasta principios de 2016. Los informes preliminares sugieren que la tasa y el patrón de falla y las tasas de necrosis, vasculopatía y complicaciones neurológicas severas, eran equivalentes a la terapia con fotones ${ }^{63}$. Tambien se observó que cuando se corrige la distribución de la dosis de radiación en el cerebro normal, aquellos tratados con RT de protones no tuvieron cambios en los puntajes académicos (lectura y matemáticas) en comparación con los pacientes tratados con RT de fotones, quienes tuvieron puntajes más bajos ${ }^{64}$. Desde 2016, se dispone de un generación, llamada de escaneo con haz de lápiz. La ventaja de este método de segunda generación sobre la primera generación es el uso de haces pequeños, ponderados individualmente para adaptar aún más la dosis de prescripción al objetivo ${ }^{2}$.

A pesar de que ha aumentado la precisión de la RT, no se ha logrado aún reducir la dosis a los tejidos normales. Esto podría ocurrir si se considera reducir la dosis total de irradiación, que generalmente es de 50-54 Gy. Es muy difícil diseñar un estudio para reducir de manera segura la dosis de irradiación por el número limitado de pacientes y el menor número de eventos después de la $\mathrm{RT}^{2}$.

\section{Panorama}

La experiencia profesional en el diagnóstico y tratamiento del CF tiene un impacto relevante en el resultado y el pronóstico de esta patología ${ }^{65-68}$. Por esto, es importante centralizar el manejo del CF en instituciones con infraestructura de alto nivel para el manejo multidisciplinario, para lo cual se deben considerar alternativas 
como las redes multicéntricas para evaluaciones de referencia y así asegurar altos estándares de calidad del tratamiento ${ }^{69,70}$. Junto con las mejoras en infraestructura, se están realizando ensayos para desarrollar mejores tratamientos para el CF, por ejemplo, terapias dirigidas que mejoren el pronóstico en general.

\section{Terapia dirigida}

Los nuevos avances en la fisiopatología molecular del CF abrieron perspectivas en el tratamiento dirigido ${ }^{35,71}$ como el vismodegib, un inhibidor de la vía SHH clínicamente aprobado y que se ha utilizado con éxito para el tratamiento del meduloblastoma y el carcinoma basocelular, asociados a la hiperactivación de la vía $\mathrm{SHH}^{72,73}$. Sin embargo, recientemente se ha demostrado que la inhibición de la vía SHH conduce a una mayor proliferación celular y aceleración en la formación del tumor en CFA en células de ratón y humano, lo que indica que esta terapia no debe intentarse en los pacientes y refuerza la idea que debe realizarse siempre una investigación preclínica ${ }^{37}$. Otra vía para explorar es la inhibición de la vía MAPK usando trametinib, un inhibidor específico de MEK ${ }^{74,75}$, que reduce el índice proliferativo y aumenta la apoptosis de las células tumorales en cultivos de celulas tumorales de CFA de ratón y humano ${ }^{35}$. En los CFA quísticos, las terapias intraquísticas se han utilizado con resultados variables. En una revisión de 56 niños con CFA, se demostró que el IFNa intraquístico retrasa la progresión de la enfermedad y es más seguro que otras modalidades terapéuticas ${ }^{59}$. Aunque los mecanismos subyacentes a los efectos de IFNa no son claros, es probable que estén asociados con su capacidad anti-inflamatoria. La importante naturaleza inflamatoria de los CFA sugiere que las nuevas terapias destinadas a inhibir la señalización de citoquinas pueden ser relevantes. Los inhibidores de IL-6 o IL-1, disponibles para otras indicacio- nes, han mostrado resultados esperanzadores. En un estudio reciente, se evidenciaron resultados prometedores en el tratamiento de CFA quístico, mediante la administración sistémica de inhibidores de IL- $6{ }^{76}$. Adicionalmente, el uso de inhibidores de Programmed Death-ligand 1 (PD-1) puede ser relevante en CFA y requerir una evaluación preclínica ${ }^{2}$.

\section{Cirugía y RT}

La RT externa es eficiente para controlar y prevenir la progresión y las recurrencias 77 . Debido a sus características físicas, la terapia con haz de protones ofrece ventajas sobre la irradiación de fotones en términos de preservar el tejido circundante, lo que disminuye el riesgo de secuelas. Sin embargo, se necesitan estudios sobre el resultado a largo plazo después de la terapia con haz de protones para probar esta hipótesis. Hay un debate sobre si la RT del tumor residual después de una resección incompleta, debe realizarse inmediatamente después de la cirugía o en el momento de su progresión. Es muy dificil realizar un estudio prospectivo aleatorizado para poder responder esta pregunta ${ }^{78}$.

\section{Conclusión}

Los esfuerzos futuros para mejorar el pronóstico, los resultados y la calidad de vida en pacientes con $\mathrm{CF}$, deben centrarse en mejorar nuestra comprensión de la fisiopatología molecular, con la perspectiva de desarrollar terapias efectivas dirigidas contra la progresión del tumor y del compromiso hipotalámico. También se requiere entender mejor las estrategias terapéuticas quirúrgicas y de RT, con el objetivo de disminuir el daño del eje hipotálamo-hipófisis y ofrecer un soporte multidisciplinario para minimizar las secuelas endocrinológicas y neuropsicológicas. 


\section{Referencias}

1. Buslei R, Rushing EJ, et al. Craniopharyngioma. En: Louis DN, Ohgaki H, Wiestler OD, Cavenee WK, editors. WHO Classification of Tumours of the Central Nervous System. Revised 4th ed. France: International Agency for Research on Cancer; 2016. 328 p.

2. Müller HL, Merchant TE, Warmuth-Metz M, Martinez-Barbera JP, Puget S. Craniopharyngioma. Nat Rev Dis Primers [Internet]. 2019;5:75 https://doi. org/10.1038/s41572-019-0133-9

3. Bunin GR, Surawicz TS, Witman PA, Preston-Martin S, Davis F, Bruner M. The descriptive epidemiology of craniopharyngioma. J. Neurosurg [Internet]. 1998; 89:547-551. https://doi.org/10.3171/ jns.1998.89.4.0547

4. Olsson DS, Andersson E, Bryngelsson IL, Nilsson AG, Johannsson G. Excess mortality and morbidity in patients with craniopharyngioma, especially in patients with childhood onset: a population- based study in Sweden. J. Clin. Endocrinol. Metab [Internet]. 2015;100:467-474. https://doi. org/10.1210/jc.2014-3525

5. Zacharia BE, Bruce SS, Goldstein H, Malone HR, Neugut Al, Bruce J. Incidence, treatment and survival of patients with craniopharyngioma in the Surveillance, Epidemiology and End Results program. Neuro Oncol [Internet]. 2012;14:1070-1078. https://doi.org/10.1093/neuonc/nos142

6. Makino K, Nakamura H, Yano S, Kuratsu J, Kumamoto Brain Tumor Group. Population- based epidemiological study of primary intracranial tumors in childhood. Childs Nerv. Syst [Internet]. 2010;26:1029-1034. https://doi.org/10.1007/ s00381-010-1126-x

7. Rosemberg S, Fujiwara D. Epidemiology of pediatric tumors of the nervous system according to the WHO 2000 classification: a report of 1,195 cases from a single institution. Childs Nerv. Syst [Internet]. 2005;21:940-944. https://doi.org/10.1007/ s00381-005-1181-x

8. Nielsen EH, Feldt-Rasmussen $U$, Poulsgaard L, Kristensen LO, Astrup J, Jørgensen JO, et al. Incidence of craniopharyngioma in Denmark $(n=189)$ and estimated world incidence of craniopharyngioma in children and adults. J. Neurooncol [Internet]. 2011;104:755-763. https://doi.org/10.1007/ s11060-011-0540-6

9. Larkin SJ, Ansorge O. Pathology and pathogenesis of craniopharyngiomas. Pituitary [Internet]. 2013;16:9-17. https://doi.org/10.1007/s11102-0120418-4
10. Muller-Scholden J, Lehrnbecher T, Müller HL, Bensch J, Hengen RH, Sörensen $\mathrm{N}$, et al. Radical surgery in a neonate with craniopharyngioma. Report of a case. Pediatr. Neurosurg [Internet]. 2000;33:265-269. https://doi. org/10.1159/000055967

11. Bailey W, Freidenberg GR, James HE, Hesselink JR, Jones KL. Prenatal diagnosis of a craniopharyngioma using ultrasonography and magnetic resonance imaging. Prenat. Diagn [Internet]. 1990;10:623629. https://doi.org/10.1002/pd.1970101002

12. Chentli F, Belhimer F, Kessaci F, Mansouri, B. Congenital craniopharyngioma: a case report and literature review. J. Pediatr. Endocrinol. Metab [Internet]. 2012; 25:1181-1183. https://doi. org/10.1515/jpem-2012-0251

13. Crotty TB, Scheithauer BW, Young WF, Davis DH, Shaw EG, Miller GM, et al. Papillary craniopharyngioma: a clinicopathological study of 48 cases. J. Neurosurg [Internet]. 1995;83:206-214. https:// doi.org/10.3171/jns.1995.83.2.0206

14. Karavitaki N, Wass J. Craniopharyngiomas. Endocrinol Metab Clin N Am [Internet]. 2008;37:173193. https://doi.org/10.1016/j.ecl.2007.10.012

15. Garnett MR, Puget S, Grill J, Sainte- Rose C. Craniopharyngioma. Orphanet Journal of Rare Diseases [Internet]. 2007;2:18. https://doi. org/10.1186/1750-1172-2-18

16. Boch AL, van Effenterre, R, Kujas M. Craniopharyngiomas in two consanguineous siblings: case report. Neurosurgery [Internet]. 1997;41:1185-1187. https://doi.org/10.1097/00006123-19971100000031

17. Green AL, Yeh JS, Dias PS. Craniopharyngioma in a mother and daughter. Acta Neurochir [Internet]. 2002;144:403-404. https://doi.org/10.1007/ s007010200058

18. Pereira AM, Schmid EM, Schutte PJ, Voormolen $\mathrm{JH}$, Biermasz NR, van Thiel SW, et al. High prevalence of long- term cardiovascular, neurological and psychosocial morbidity after treatment for craniopharyngioma. Clin. Endocrinol [Internet]. 2005;62:197-204. https://doi.org/10.1111/j.13652265.2004.02196.x

19. Muller HL, Bueb K, Bartels U, Roth C, Harz $\mathrm{K}$, Graf N, et al. Obesity after childhood craniopharyngioma - German multicenter study on pre- operative risk factors and quality of life. Klin. Padiatr [Internet]. 2001;13:244-249. https://doi. org/10.1055/s-2001-16855

20. Poretti A, Grotzer MA, Ribi K, Schonle E, Boltshauser $\mathrm{E}$. Outcome of craniopharyngioma in children: long- term complications and quality of life. 
Dev. Med. Child Neurol [Internet]. 2004;46:220229. https://doi.org/10.1017/s0012162204000374

21. Visser J, Hukin J, Sargent $M$, Steinbok P, Goddard K, Fryer C. Late mortality in pediatric patients with craniopharyngioma. J. Neurooncol [Internet]. 2010; 100:105-111 (2010). https://doi. org/10.1007/s11060-010-0145-5

22. Hoffmann, A. et al. Fusiform dilatation of the internal carotid artery in childhood- onset craniopharyngioma: multicenter study on incidence and long- term outcome. Pituitary [Internet]. 2016;19,422-428.

23. Wijnen $M$, Olsson DS, van der Heuvel-Eibrink MM, Hammarstrand C, Lanssen J, et al. Excess morbidity and mortality in patients with craniopharyngioma: a hospital- based retrospective cohort study. Eur. J. Endocrinol [Internet]. 2018; 178:93-102. https://doi.org/10.1530/eje-17-0707

24. Wijnen $M$, Olsson DS, van der Heuvel-Eibrink MM, Hammarstrand C, Lanssen J, et al. The metabolic syndrome and its components in 178 patients treated for craniopharyngioma after 16 years of follow- up. Eur. J. Endocrinol [Internet]. 2018;178:11-22. https://doi.org/10.1530/eje-17-0387

25. Wijnen $M$, van der Heuvel-Eibrink $M M$, Lanssen J, Castman-Berrevoets CE, Michiels EM, et al. Very long- term sequelae of craniopharyngioma. Eur. J. Endocrinol [Internet]. 2017;176:755-767. https:// doi.org/10.1530/eje-17-0044

26. Holmer H, Popovic V, Ekman B, Follin C, Siversson $A B$, Esfurth EM. Hypothalamic involvement and insufficient sex steroid supplementation are associated with low bone mineral density in women with childhood onset craniopharyngioma. Eur. J. Endocrinol [Internet]. 2011;165:25-31. https://doi.org/10.1530/eje-11-0229

27. Hoffmann A, Bootsveld K, Gebhardt Um Daubenbüchel AM, Sterkenburg AS, Müller HL. Nonalcoholic fatty liver disease and fatigue in long- term survivors of childhood- onset craniopharyngioma. Eur. J. Endocrinol [Internet]. 2015; 173:389-397. https://doi.org/10.1530/eje-15-0422

28. Heinks K, De Schutter-Nüsse C, Boekhoff $S$, Bogusz A, Zhu J, Peng J, et al. Periostin concentrations in childhoodonset craniopharyngioma patients. J. Endocrinol. Invest [Internet]. 2019;42:815-824. https://doi.org/10.1007/s40618018-0987-9

29. Erfurth EM, Holmer H, Fjalldal SB. Mortality and morbidity in adult craniopharyngioma. Pituitary [Internet] 2013;16:46-55. https://doi.org/10.1007/ s11102-012-0428-2

30. Sekine S, Shibata T, Kokubu A, Morishita Y,
Noguchi M, Nakanishi Y, et al. Craniopharyngiomas of adamantinomatous type harbor beta- catenin gene mutations. Am. J. Pathol [Internet]. 2002;161:1997-2001. https://doi.org/10.1016/ s0002-9440(10)64477-x

31. Buslei R, Nolde M, Hofmann B, Meissner S, Eyupoglu IY, Siebzehnrübl F, et al. Common mutations of $\beta$ - catenin in adamantinomatous craniopharyngiomas but not in other tumours originating from the sellar region. Acta Neuropathol [Internet]. 2005;109:589-97. https://doi.org/10.1007/s00401005-1004-X

32. Brastianos PK, Taylor-Weiner A, Manley PE, Jones RT, Dias-Santagata D, Thorner AR, et al. Exome sequencing identifies BRAF mutations in papillary craniopharyngiomas. Nat. Genet [Internet]. 2014;46:161-165. https://doi.org/10.1038/ng.2868

33. Hölsken A, Buchfelder M, Fahlbusch, Blümcke I, Buslei R. Tumour cell migration in adamantinomatous craniopharyngiomas is promoted by activated Wnt-signalling. Acta Neuropathol [Internet]. 2010;119:631-639. https://doi.org/10.1007/s00401010-0642-9

34. Gaston-Massuet C, Andoniadoua CL, Signore M, Jayakodya SA, Charolidi N, Kyeyune R, et al. Increased Wingless (Wnt) signaling in pituitary progenitor/stem cells gives rise to pituitary tumors in mice and humans. Proc Natl Acad Sci USA [Internet]. 2011; 108(28):11282-7. https://doi.org/10.1073/ pnas. 1101553108

35. Apps JR, Carreño G, González-Meljem JM, Haston S, Guiho R, Cooper JE, et al. Tumour compartment transcriptomics demonstrates the activation of inflammatory and odontogenic programmes in human adamantinomatous craniopharyngioma and identifies the MAPK/ERK pathway as a novel therapeutic target. Acta Neuropathol [Internet]. 2018;135(5):757-777. https:// doi.org/10.1007/s00401-018-1830-2

36. Andoniadou CL, Gaston-Massuet C, Reddy $\mathrm{R}$, Schneider RP, Blasco MA, Le Tissier P, et al. Identification of novel pathways involved in the pathogenesis of human adamantinomatous craniopharyngioma. Acta Neuropathol [Internet]. 2012;124(2):259-271. https://doi.org/10.1007/ s00401-012-0957-9

37. Carreno G, Boult JKR, Apps J, González-Meljem JM, Haston S, Guiho R, Stache C, et al. SHH pathway inhibition is protumourigenic in adamantinomatous craniopharyngioma. Endocr. Relat. Cancer [Internet]. 2019;26(3):355-366. https:// doi.org/10.1530/erc-18-0538

38. Goschzik, T, Gwaae M, Dreschmann V, Gebhardt 
U, Wang L, Yamaguchi S, et al. Genomic alterations of adamantinomatous and papillary craniopharyngioma. J. Neuropathol. Exp. Neurol [Internet]. 2017;76(2):126-134. https://doi.org/10.1093/jnen/ nlw116

39. Holsken A, Sill M, Merkle J, Schweizer L, Buchfelder M, Flitsch J, et al. Adamantinomatous and papillary craniopharyngiomas are characterized by distinct epigenomic as well as mutational and transcriptomic profiles. Acta neuropathol Commun [Internet]. 2016;4:20. https://doi.org/10.1186/ s40478-016-0287-6

40. Haston S, Pozzi S, Carreno G, Manshaer S, Paousopoloulos L, González-Meljem JM, et al. MAPK pathway control of stem cell proliferation and differentiation in the embryonic pituitary provides insights into the pathogenesis of papillary craniopharyngioma. Development [Internet]. 2017;144(12):2141-2152. https://doi.org/10.1242/ dev.150490

41. Müller HL, Emser A, Faldum A, Bruhnken G, Etavard-Gorris N, Gebhardt U, et al. Longitudinal study on growth and body mass index before and after diagnosis of childhood craniopharyngioma. J. Clin. Endocrinol. Metab [Internet]. 2004; 89(7):3298-3305. https://doi.org/10.1210/jc.2003031751

42. Hoffmann A, Boekhoff S, Gebhardt U, Sterkenburg AS, Daubenbüchek AMM, Eveslage $M$, et al. History before diagnosis in childhood craniopharyngioma: associations with initial presentation and long-term prognosis. Eur J Endocrinol [Internet]. 2015;173(6):853-862. https://doi.org/10.1530/eje15-0709

43. Prieto R, Pascual JM, Barrios L. Optic chiasm distortions caused by craniopharyngiomas: clinical and magnetic resonance imaging correlation and influence on visual outcome. World Neurosurg [Internet]. 2015;83(4):500-529. https://doi. org/10.1016/j.wneu.2014.10.002

44. Müller HL. Childhood Craniopharyngioma. Horm Res [Internet]. 2008;69:193-202. https:// doi.org/10.1159/000113019

45. Zhou L, Luo L, Xu J, Chen J, Jiang S, Cai B, et al. Craniopharyngiomas in the posterior fossa: a rare subgroup, diagnosis, management and outcomes. J Neurol Neurosurg Psychiatry [Internet]. 2009;80(10):1150-1155. https://doi.org/10.1136/ jnnp.2008.149088

46. Elliott RE, Jane JAJr, Wisoff JH. Surgical management of craniopharyngiomas in children: meta- analysis and comparison of transcranial and transsphenoidal approaches. Neurosur- gery [Internet]. 2011;69(3):630-643. https://doi. org/10.1227/neu.obo13e31821a872d

47. Muller, H. L. Childhood craniopharyngioma. Recent advances in diagnosis, treatment and followup. Horm. Res [Internet]. 2008;69(4):193-202. https://doi.org/10.1159/000113019

48. Hoffman HJ, De Silva M, Humphreys RP, Drake JM, Smith ML, Blaser SI. Aggressive surgical management of craniopharyngiomas in children. J. Neurosurg [Internet]. 1992;76(1):47-52. https:// doi.org/10.3171/jns.1992.76.1.0047

49. Roth CL, Gebhardt U, Müller HL. Appetite-regulating hormone changes in patients with craniopharyngioma. Obesity [Internet].2011;19(1):3642. https://doi.org/10.1038/oby.2010.80

50. Kilday JP, Bartels U, Huang A, Barron M, Shago $M$, Mistry $M$, et al. Favorable survival and metabolic outcome for children with diencephalic syndrome using a radiation- sparing approach. J. Neurooncol [Internet]. 2014; 116(1):195-204. https://doi.org/10.1007/s11060-013-1284-2

51. Hoffmann A, Gebhardt U, Sterkenburg AS, Warmuth- Metz M, Müller HL. Diencephalic syndrome in childhood craniopharyngioma- results of german multicenter studies on 485 long- term survivors of childhood craniopharyngioma. J. Clin. Endocrinol. Metab [Internet]. 2014;99(11):39723977. https://doi.org/10.1210/jc.2014-1680

52. Rossi A, Cama A, Consales A, Gandolfo C, Garrè $M L$, Milanaccio $C$, et al. Neuroimaging of pediatric craniopharyngiomas: a pictorial essay. J. Pediatr. Endocrinol. Metab. 2006; 19(Suppl 1):299-319. PMID: 16700305

53. Hoffmann A, Brentrup A, Muller HL. First report on spinal metastasis in childhood- onset craniopharyngioma. J. Neurooncol [Internet]. 2016;129(1):193-194. https://doi.org/10.1007/ s11060-016-2160-7

54. Kordes $\mathrm{U}$, Flitsch $\mathrm{CH}$, Goebell E, Rchwarz $R$, Herberhold $A$, von Bueren $A O$, et al. Ectopic craniopharyngioma. Klin. Padiatr [Internet]. 2011;223(3):176-177. https://doi.org/10.1055/s-0031-1273743

55. Warmuth-Metz M, GnekowAK, Muller H, Solymosi L. Differential diagnosis of suprasellar tumors in children. Klin Padiatr [Internet]. 2004;216(6):323330. https://doi.org/10.1055/s-2004-832358

56. Prieto R, Pascual JM, Hofecker V, Winter E, Castro-Dufourny I, Carrasco R, et al. Craniopharyngioma adherence: a reappraisal of the evidence. Neurosurg. Rev [Internet]. 2018;43:453-472. https:// doi.org/10.1007/s10143-018-1010-9.

57. Hill TK, Baine MJ, Verma V, Alam M, Lyden ER, Lin 
C, et al. Patterns of care in pediatric craniopharyngioma: outcomes following definitive radiotherapy. Anticancer Res [Internet]. 2019;39(2):803807. https://doi.org/10.21873/anticanres.13178

58. Cavalheiro S, Di Rocco C, Valenzuela S, Dastoli PA, Tamburrini G, Massimi L, et al. Craniopharyngiomas: intratumoral chemotherapy with interferon-alpha: a multicenter preliminary study with 60 cases. Neurosurg Focus [Internet]. 2010; 28(4):E12. https://doi.org/10.3171/2010.1.focuso9310

59. Kilday JP, Caldarelli M, Massimi L, Chen RH, Lee $Y Y$, Liang $M$, et al. Intracystic interferon-alpha in pediatric 434 craniopharyngioma patients: an international multicenter assessment on behalf of SIOPE and 435 ISPN. Neuro Oncol [Internet]. 2017;19(10):1398-1407. https://doi.org/10.1093/ neuonc/nox056

60. Zhang S, Fang Y, Cai BW, Xu JG, You C. Intracystic bleomycin for cystic craniopharyngiomas in children. Cochrane Database Syst [Internet]. 2016; 7(7):CDoo8890. https://doi.org/10.1002/14651858. cdoo8890.pub4

61. Adeberg S, Harrabi SB, Bougatf N, Verma V, Windisch P, Bernhardt D, et al. Dosimetric Comparison of Proton Radiation Therapy, Volumetric Modulated Arc Therapy, and Three-Dimensional Conformal Radiotherapy Based on Intracranial Tumor Location. Cancers [Internet]. 2018;10(11):401. https://doi.org/10.3390/cancers10110401

62. Müller HL. Hypothalamic involvement in craniopharyngioma - implications for surgical, radiooncological, and molecularly targeted treatment strategies. Pediatr. Blood. Cancer [Internet]. 2018;65(5):e26936. https://doi.org/10.1002/ pbc. 26936

63. Merchant TE, Hua C-H, Sabin ND, Wu S, Khan RB, Indelicato, et al. Necrosis, vasculopathy, and neurological complications after proton therapy for childhood craniopharyngioma: Results from a prospective trial and a photon cohort comparison. Int J Rad Biol Oncol Phys [Internet]. 2016;96(2):S12- S121. https://doi.org/10.1016/j. ijrobp.2016.06.294

64. Merchant T, Indelicato D, Hua C, Wu S, Conklin $430 \mathrm{H}$. Comparison of academic scores after proton and photon therapy in children and young adults with craniopharyngioma. Pediatr Blood Cancer [Internet]. 2017;64:e2677.

65. Muller HL, Gebhardt U, Faldum A, Warmuth-Metz M, Pietsch T, Pohl F, et al. Xanthogranuloma, Rathke's cyst, and childhood craniopharyngioma: results of prospective multinational studies of children and adolescents with rare sellar malformations. J. Clin. Endocrinol. Metab [Internet]. 2012;97(11):3935-3943. https://doi. org/10.1210/jc.2012-2069

66. Muller HL, Gebhardt U, Teske C, Faldum A, Zwiener I, Warmuth-Metz $M$, et al. Post- operative hypothalamic lesions and obesity in childhood craniopharyngioma: results of the multinational prospective trial KRANIOPHARYNGEOM 2000 after 3-year follow- up. Eur. J. Endocrinol [Internet]. 2011;165(1):17-24. https://doi.org/10.1530/eje-110158

67. Hankinson TC, Palmeri NO, Williams SA, Torok MR, Serramo CA, Foreman NK, et al. Patterns of care for craniopharyngioma: survey of members of the American Association of Neurological Surgeons. Pediatr. Neurosurg [Internet]. 2013; 49(3):131-136. https://doi.org/10.1159/000357783

68. Schwartz TH. A role for centers of excellence in transsphenoidal surgery. World Neurosurg [Internet]. 2013;80(3):270-271. https://doi.org/10.1016/j. wneu.2012.11.019

69. Tallen G, Resch A, Calaminus G, Wiener A, Leiss $U$, Pletschko T, et al. Strategies to improve the quality of survival for childhood brain tumour survivors. Eur. J. Paediatr. Neurol [Internet]. 2015;19(6):619-639. https://doi.org/10.1016/j. ejpn.2015.07.011

70. Müller HL, Reichel J, Boekhoff S, Warmuth-Metz $M$, Eveslage M, Peng J, et al. Low concordance between surgical and radiological assessment of degree of resection and treatment- related hypothalamic damage: results of KRANIOPHARYNGEOM 2007. Pituitary [Internet]. 2018;21(4),371-378. https://doi.org/10.1007/s11102-018-0883-5

71. Gump JM, Donson AM, Birks DK, Amani VM, Rao $\mathrm{KK}, \mathrm{Griesinger} A M$, et al. Identification of targets for rational pharmacological therapy in childhood craniopharyngioma. Acta Neuropathol. Commun [Internet]. 2015;3:30. https://doi.org/10.1186/ s40478-015-0211-5

72. Rimkus TK, Carpenter RL, Qasem S, Chan M, Lo HW. Targeting the sonic hedgehog signaling pathway: review of Smoothened and GLI inhibitors. Cancers [Internet]. 2016;8(2):E22. https://doi. org/10.3390/cancers 8020022

73. Sekulic A, Migden MR, Basset-Seguin N, Garbe $C$, Geiesrich A, Lao CD, et al. Long- term safety and efficacy of vismodegib in patients with advanced basal cell carcinoma: final update of the pivotal ERIVANCE BCC study. BMC Cancer [Internet]. 2017;17(1):332. https://doi.org/10.1186/s12885-0173286-5 
74. Miller C, Guillaume D, Dusenbery K, Clark HB, Moertel $C$. Report of effective trametinib therapy in 2 children with progressive hypothalamic optic pathway pilocytic astrocytoma: documentation of volumetric response. J. Neurosurg. Pediatr [Internet]. 2017;19(3):319-324. https://doi. org/10.3171/2016.9.peds16328

75. Robert C, Flaherty KT, Hersey P, Nathan PD, Garbe C, Milhem MM, et al. METRIC phase III study: efficacy of trametinib ( $T$ ), a potent and selective mek inhibitor (MEKi), in progression- free survival (PFS) and overall survival (OS), compared with chemotherapy $(C)$ in patients (pts) with BRAF$\mathrm{V} 600 / \mathrm{k}$ mutant advanced or metastatic melanoma (MM). J. Clin. Oncol [Internet]. 2012;30(18 Suppl) LBA8509. https://doi.org/10.1200/jco.2012.30.18_ suppl.lba8509
76. Grob S, Mirsky DM, Donson AM, Dahl N, Foreman NK, Hoffman LM, et al. Targeting IL-6 is a potential treatment for primary cystic craniopharyngioma. Front. Oncol [Internet]. 2019;9:791. https:// dx.doi.org/10.3389\%2Ffonc.2019.00791

77. Ismail D, O'Connell MA, Zacharin MR. Dexamphetamine use for management of obesity and hypersomnolence following hypothalamic injury. J. Pediatr. Endocrinol. Metab [Internet]. 2006;19(2):129-134. https://doi.org/10.1515/ jpem.2006.19.2.129

78. Eveslage $M$, Calaminus $G$, Warmuth-Metz $M$, Kortmann R, Pohl F, Timmermann B, et al. The postoperative quality of life in children and adolescents with craniopharyngioma. Dtsch Arztebl. Int [Internet]. 2019;116(18);321-328. https://dx. doi.org/10.3238\%2Farztebl.2019.0321 